\title{
Ordering of Nanoparticles along Concentric Nanoings Observed in Al-Cu-Fe Alloy
}

\author{
Chunfei $\mathrm{Li}^{1}$, Breanne Kothe ${ }^{1}$, Matthew Caputo ${ }^{2}$, and Helen Hampikian ${ }^{1}$ \\ ${ }^{1}$ Clarion University, Clarion, PA 16214, USA \\ ${ }^{2}$ Youngstown State University, Youngstown, OH 44555, USA
}

This paper reports the natural ordering of nanoparticles along concentric rings in Al-Cu-Fe alloy. Al-CuFe alloy with nominal composition in the vicinity of $\mathrm{Al}_{65} \mathrm{Cu}_{20} \mathrm{Fe}_{15}$ has drawn intensive interest because of its capability to form stable icosahedral quasicrystalline phase [1]. It has been revealed that, the asprepared alloys of this composition by arc melting consist of $\beta$-phase with composition of $\mathrm{AlFe}(\mathrm{Cu})$ and cubic crystal structure [2]. Further, it has been reported that the $\beta$-phase is of spherical morphology and the surface of the spheres are decorated with concentric rings [2-3]. The diameters of the most inner ring was found to be in the order of $1 \mu \mathrm{m}$, while that of the outer-most ring is in the order of $5 \mu \mathrm{m}$. The spacing between adjacent rings is in the order of $0.5 \mu \mathrm{m}$ in the inner region. Our recent studies further revealed that nanoparticles can use these concentric rings as template and form natural patterns, which is reported in the present paper.

Alloys were prepared by arc melting pure $\mathrm{Al}, \mathrm{Cu}$, and $\mathrm{Fe}$ with a nominal atomic percentage composition of $\mathrm{Al}_{65} \mathrm{Cu}_{25} \mathrm{Fe}_{15}{ }^{4}$. A TESCAN Vega-3 XMU Scanning Electron Microscope (SEM) was used to examine the surface morphology of the as-prepared alloy.

A typical SEM image of the concentric ring is shown in Fig. 1(a), which has been reported previously [2-3]. The new finding of the present work is shown in Figs. 1(b) and 1(c), where nanoparticles of several ten to hundred nanometer size are observed to be on the surface of the spherical particles. Further, it was found that these particles use the concentric rings as template to form concentric circles. It is interesting to note that, in Fig. 1(b), the concentric rings have approximately equal spacing for extended region and the ring pattern of nanoparticles continue all the way to the center. Single nanoparticles line up along the ring in Fig. 1(b). In Fig. 1(c), there are two obvious inner rings that stand out from the rest. Multiple nanoparticles agglomerate in the width direction of the rings. The inner ring has diameter of one micrometer order and the spacing between the first and second rings is in the order of 0.5 micrometer. Moving further out from the second ring, there are multiple rings made up of single nanoparticles much like the case of Fig. 1(b). The feature of rings in Fig. 1(c) is in consistent with the typical concentric nanorings reported so far [3].

It should be pointed out that the probability of finding the structures shown in Figs. 1(b) and (c) is rare. Detailed experimental studies, such as the side view observation of the nanoparticle concentric rings and an Energy Dispersive X-ray Spectroscopy (EDS) analysis of the nanoparticles relative to the sphere substrate is planned, to have a better understanding about the formation mechanism for such feature. There are some observation that give us hint about this. Fig. 1(e) shows a sphere observed in this alloy. There are two areas with bright and dark SEM contrast. Further, concentric rings are observed on the sphere. Based on such observation, it is suggested that the spherical shape of the $\beta$-phase forms due to the surface tension effect of the liquid before its crystallization. At that point, the liquid surface is covered with solid oxide probably due to the residual air in the arc melting chamber. As the liquid crystallizes to $\beta$-phase, the concentric rings is formed to minimize the surface energy. As a result, the 
surface oxide was broken, the fragments follow the template of the concentric ring and form circular patterns.

\section{References}

[1] A.P. Tsai, A. Inoue, and T. Masumoto, Jpn. J. Appl. Phys. 26, 505 (1987).

[2] K. Balzuweits, H. Meekesa, G. Van Tendeloob, and J. L. De Boerc (1993) On the Relationship Between Morphology, Composition and Structure of Al-Cu-Fe Crystals and Quasicrystals. Philos Mag B 67: 513-532

[3] C. Li, L. Wang, H. Hampikian, M. Bair, A. Baker, M. Hua, Q. Wang, and D. Li, "Concentric Nano Ring Observed on Al-Cu-Fe Microsphere", Appl. Phys. Lett. , 108 (2016) 223105-1-4.

[4] The authors acknowledge funding from the National Science Foundation (DMR-1461607).
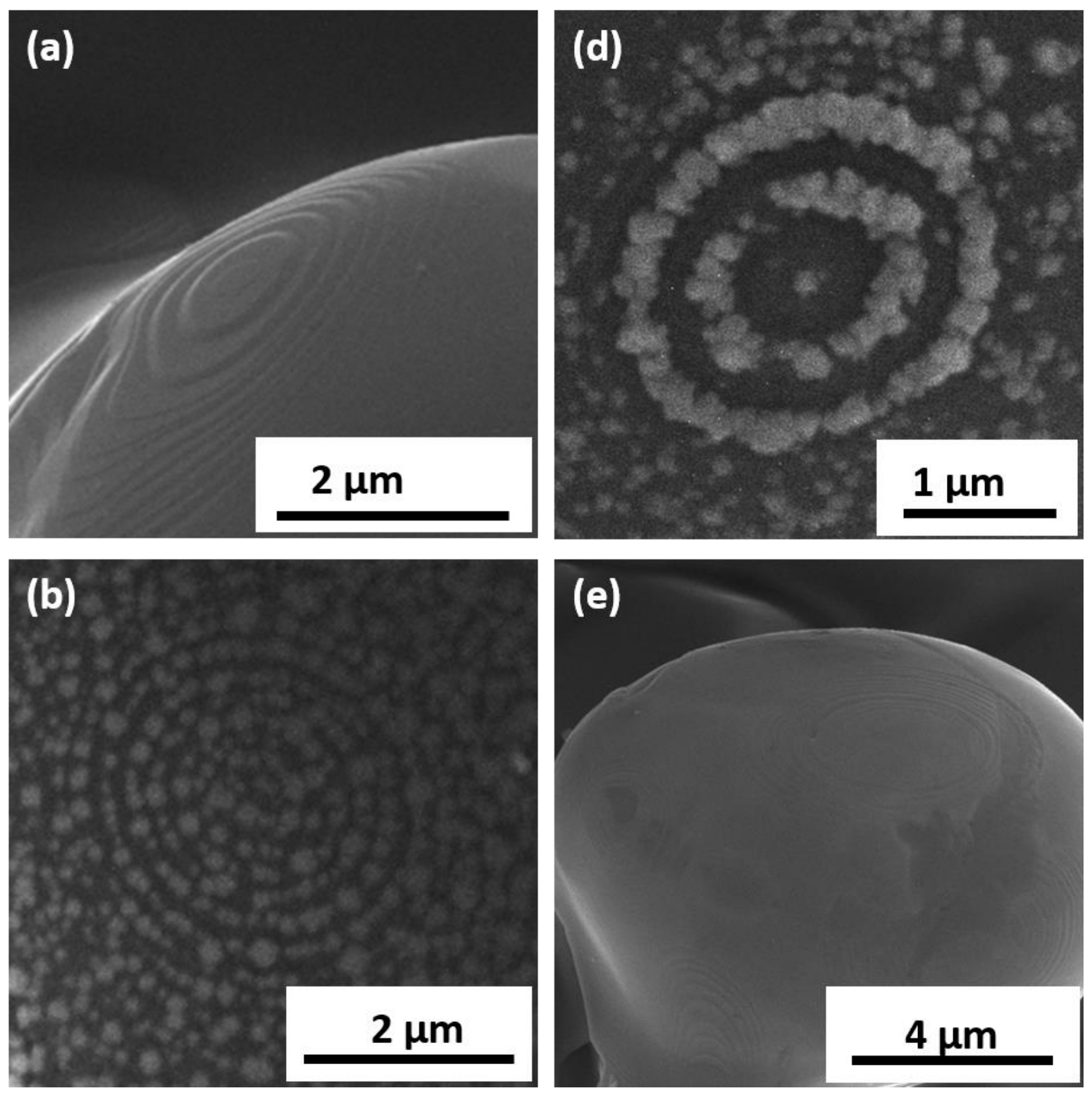

Figure 1. SEM images showing representative concentric rings (a), nanoparticles ordered along concentric rings (b) and (c), and a sphere with the precursors of nanopartilces. 